Panel Survey Approach

\title{
Panel Survey Approach to Measuring Season Ticket Use
}

\author{
Tomás Ruiz, Technical University of Valencia
}

\begin{abstract}
This article presents the results of a panel survey designed to determine season travel ticket use in the metropolitan area of Valencia, Spain. The procedure consisted of surveying a randomly selected panel of season ticket users within a given study area in spring 2002, fall 2002, and late winter 2002-2003. A total of 143 respondents were recruited by phone calls from existing users of season tickets. Each respondent was asked to complete a four-day trip diary in each survey. Only 53 panelists gave a valid response in the second survey wave. Of the panelists who participated in the three waves, 31 provided valid responses. Results revealed a higher increase of season ticket use among those 45 years and older, followed by male users, travelers with more than one car at home, and those with car availability.
\end{abstract}

\section{Background}

Season travel tickets (STs) are used to change people's travel behavior through personalized marketing - a mix of persuasion and information (Blackman 2003). They also provide a mechanism for making better use of spare capacity on the public transportation network.

The recently founded Metropolitan Transportation Authority (eTM) of Valencia, Spain, sponsors the STs. The ticket permits unlimited use of all urban and metropolitan public transportation modes during 30 consecutive days. The City of Valencia operates EMT, the urban bus network. Metrobus is the metropolitan 
bus network; FGV, the public railway company, operates both in the urban and metropolitan areas. RENFE, the national railway network that operates as a metropolitan system in the Valencia area, is not included. The eTM must compensate economically with public transportation companies for the loss in fare revenue caused by use of ST. At the time of this research, ST use was not fully automatically controlled. Nonpanel periodic surveys were usually done to assess ST use.

The ST is a personal travel ticket. Travelers who use it are included in two databases maintained by the FGV. Demographic and socioeconomic data are collected from every new ST user. An identification card containing a unique identification number (ID) is given to new users. Whenever a traveler renews an ST, his or her ID appears in the database as a valid ST. In 30 days the ST becomes invalid, unless the user renews it again.

The ST type-B (ST-B), which is only valid for traveling between metropolitan towns and the City of Valencia, was chosen as the study test. Results were utilized to determine the economic compensation that each public transportation company must realize by use of the ST-B, according to an established agreement between eTM and public transportation companies.

\section{Panel Survey Approach}

Public perceptions and attitudes of public transportation users can be investigated using several survey techniques. For example, a public participation process was used in Phoenix, Arizona, for the planning and implementation of a neighborhood circulator service (Miller 2002). A similar approach was utilized in Portland, Oregon, to develop a light rail project (Watts and Ontiveros 2001).

The study of public transportation of special user groups is relevant for developing user profiles. Knutsson (2003) compared the quality of the Swedish Special Transport Services with present-day public transportation standards. User groups were also considered in analyses about new fare systems (Gründel 2002) and studies of specific public transportation modes (Polzin and Baltes 2002).

The study of public transportation ticket usage is necessary in several situations. For instance, there is a need for information about public transportation ticket use to assist in the allocation of farebox revenue to operators within a privatized public transportation scheme (Richardson et al. 1998). The relationship between car ownership and STs has been studied by Axhausen et al. (2001) to understand 
how individuals' long-term decisions influence their daily or short-term behavior. In both cases, cross-sectional surveys were carried out.

The study of ST use variation can be examined using multiperiod surveys. Two types of multiperiod surveys are common (Purvis and Ruiz 2003): repeated crosssection surveys (same survey at intervals in time applied to different respondents), and panel surveys (same survey at intervals in time applied to the same respondents). Measuring ST use with nonpanel periodical surveys provides limited information. Cross-sectional or repeated cross-sectional surveys only provide results in the form of slight increases or declines in ST use. This aggregate data cannot be used to identify who is increasing or reducing their mobility in public transportation.

Panel surveys, in which the same observational units are surveyed at several points in time, offer a number of important advantages over other periodic and nonperiodic mobility collection methods. Moser and Kalton (1979) pointed out that panel data are more accurate than repeated cross-sections data with the same sample size when the differences between two measures are analyzed. Duncan et al. (1987) also argued that the best way to investigate changes in attitudinal characteristics is by using panel surveys. In addition, Kitamura (1990) completed this set of advantages, indicating that repeated surveys of the same respondents make it possible to control explicative factors not observed. The identification of cause-effect relations is easier. Also, dynamic models better predict the future mobility because longitudinal data have more information per analysis unit than nonperiodic data.

On the other hand, panel surveys have some drawbacks. Moser and Kalton (1979) indicated that it is most difficult to get a good sample because people are reluctant to be interviewed several consecutive times. Panelists' responses, or their behavior, can be influenced by the panel survey itself. This is called the "panel effect." Hensher (1986) argued that the main results can be obtained only at the end of the panel, several years after its beginning. This fact makes it difficult for panel surveys to be financially supported.

Relative to the ST-B use study approach, the panel survey offers the following advantages:

- The survey readily collects information concerning ST-B use for each trip made. Hence, route-shift effects can be identified. 
Journal of Public Transportation, Vol. 7, No. 4, 2004

- A wide range of socioeconomic characteristics of each ST-B user can be obtained. Hence, differences in ST-B use evolution among different groups of people can be examined.

- Panels help to identify components of the direct effects of transportation policy on travel behavior (Goodwin 1997).

\section{Panel Survey Design and Execution}

Initially, the panel survey to measure the use of the ST-B coincided with a crosssectional investigation of the use of ST types-A and -AJ (only valid in the City of Valencia) (Ruiz et al. 2003). Only those respondents who were ST-B users and gave valid responses were contacted again.

The following sections present a summary of the recruitment process, a description of the questionnaires used, and the initial response rates obtained. More details can be found in Ruiz et al. (2003).

\section{Recruitment of Panelists}

About two weeks before sending the precontact letter to selected respondents in spring 2002, an advertising campaign was done at bus stops, subway and railway stations, and on public transportation vehicles.

The first contact with selected respondents was carried out by means of a postcard. Since research has shown that official sponsorship is likely to increase the response rate (Richardson et al. 1995; Turley 1999), the postcard contained the letterheads of public transportation operators and the eTM. The card emphasized the main objective of the survey, the importance of respondent collaboration, and the confidentiality of the data collected. The card also announced the next phone contact.

Telephone calls were utilized both for recruiting respondents in the first wave and for recontacting them in the second and third waves (carried out in fall 2002 and late winter 2002-2003, respectively). Phone calls were also used for both the main panelists and the refreshment sample members. Interviewers made a maximum of 10 phone calls at different times during three consecutive days. If the contact failed, it was considered a nonresponse. Otherwise, the respondent was asked about the precontact letter and its content. If the respondent was willing to participate in the survey, an appointment was made for the first personal interview. 


\section{Data Collection Instruments}

The first personal interview served mainly to gather demographic and socioeconomic data about the respondent. The interviewer collected the answers and filled in forms. Next, the interviewer delivered a four-day trip diary to the respondent (Figure 1), and showed how to fill it in. The interviewer indicated the four-day period in which the respondent should use the diary and how to fill in the characteristics of all the trips made using the ST-B. Finally, another appointment was made to collect the diary. During the second personal contact, the interviewer briefly reviewed the diary to ensure that information about the four-day period was entered properly. If any deficiency was found in the diary data, the interviewer asked the respondent to resolve it. The trip diary remained the same in the three survey waves and the starting day of week was also the same for each respondent in each wave. Neither incentives nor maintenance methods were utilized.

\section{Figure 1. Sample Four-Day Trip Diary}

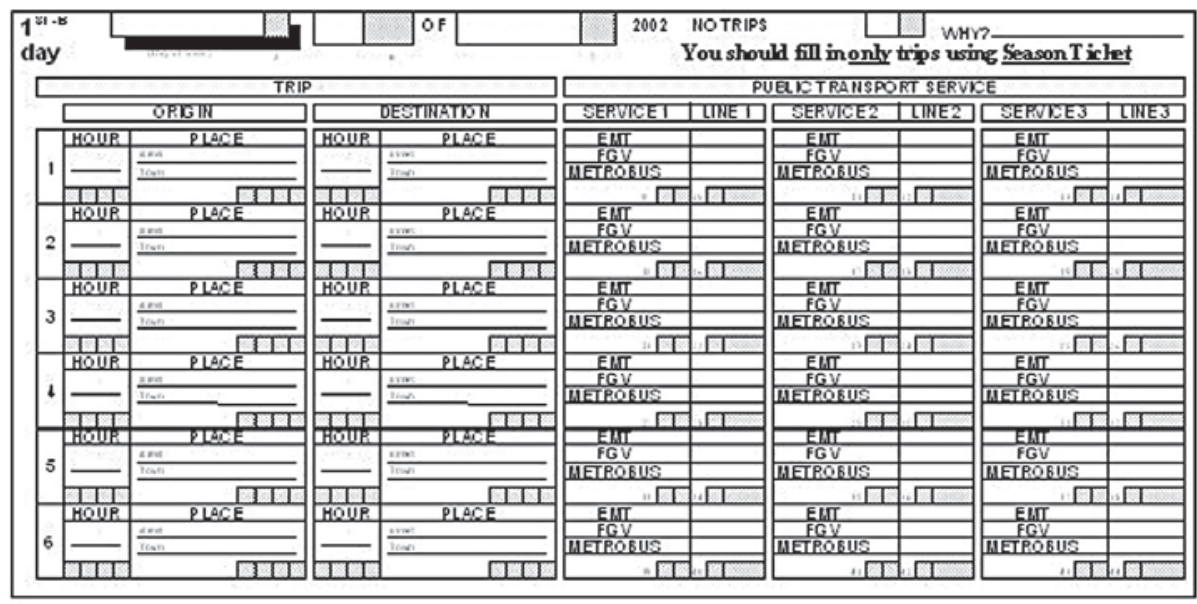




\section{Response Rates}

In spring 2002, about 291 respondents were initially selected from the database; 143 valid responses were obtained, for a response rate of 62.7 percent from the net sample size. Members of the sample from whom a response could not possibly be obtained were subtracted from the gross sample size. This type of sample loss (phone line damaged, invalid phone numbers, ST-B expired) did not affect the quality of the sample (Table 1).

Table 1. Initial Response Rate

\begin{tabular}{l|c|lc}
\hline & Responses & & Responses \\
\hline Gross sample size & 291 & Not contacted & 34 \\
Phone line damaged & 19 & Refusals & 29 \\
Invalid phone numbers & 31 & Agree to participate & 165 \\
Season travel ticket expired & 13 & Abandons & 22 \\
Net sample size & 228 & Valid responses & 143 \\
\hline & & Response rate & $62.7 \%$ \\
\hline
\end{tabular}

\section{Quality of the Data}

\section{Sample Bias}

To avoid sample bias, it is necessary to select the sample using a random procedure. The systematic selection employed in this study was not a random procedure. However, if databases are not ordered according to any variable related to ST-B use, the sample obtained is random. Besides, the data representativity might be reduced if the response rate is very low. If the majority of nonrespondents have a similar characteristic related to the variable studied, the data obtained will be biased.

In this case, several demographic and socioeconomic distributions of the sample data have been compared with the same distributions of the population of interest (valid ST-B users). Generally, small differences were found (Table 2). Female ST-B users, travelers more than 35 years old, and the employed were slightly overrepresented. 


\section{Table 2. Representativeness of the Sample}

\begin{tabular}{|l|cc|}
\hline \multicolumn{1}{|c|}{ Sex } & Sample & Population \\
\hline Men & $20 \%$ & $24 \%$ \\
Female & $80 \%$ & $76 \%$ \\
\hline
\end{tabular}

\begin{tabular}{|l|cc|}
\hline \multicolumn{1}{|c|}{ Age } & Sample & Population \\
\hline$<18$ & $5 \%$ & $9 \%$ \\
$18-25$ & $36 \%$ & $40 \%$ \\
$26-35$ & $23 \%$ & $24 \%$ \\
$>35$ & $36 \%$ & $27 \%$ \\
\hline
\end{tabular}

\begin{tabular}{|l|cc|}
\hline \multicolumn{1}{|c|}{ Activity } & Sample & Population \\
\hline Employed & $60 \%$ & $53 \%$ \\
Students & $33 \%$ & $39 \%$ \\
Others & $7 \%$ & $8 \%$ \\
\hline
\end{tabular}

\section{Panel Attrition}

Only 53 of the 143 respondents who gave valid responses in the first wave provided valid responses in the second survey wave carried out in fall 2002. This equates to a panel attrition of 63 percent. Additionally, valid responses were also obtained in the second wave from 91 new respondents, who were randomly selected from the ST-B user list. The third survey wave occurred in late winter 2002-2003. A total of 31 panelists provided valid responses in the three waves. The third wave panel attrition was reduced to 42 percent for the interviewees who participated in the first one. The number of abandonments in the refreshment sample was 41, yielding an attrition rate of 45 percent. No new respondents were selected in the third survey wave, due to budget restrictions.

Among the respondents who no longer belonged to the sampling frame were: previous respondents who were no longer valid ST owners; those who changed their residential location out of the study area; and/or those who changed to other ticket types. Thus, these individuals were not taken into account for calculating attrition rates in Table 3.

Table 3. Reasons for Panel Attrition

\begin{tabular}{l|cc|cc|cc|c}
\hline & \multicolumn{4}{|c|}{ Main Panel } & \multicolumn{2}{c}{ Refreshment } \\
Sample \\
& Second & Wave & \multicolumn{2}{|c|}{ Third Wave } & & \\
& $n$ & $\%$ & $n$ & $\%$ & $n$ & $\%$ \\
\hline Noncontacts & 52 & 36.4 & 12 & 22.6 & 22 & 24.2 \\
Not ST user or residential change & 2 & 1.4 & 2 & 3.8 & 5 & 5.5 \\
Change to other ticket type & 1 & 0.7 & 2 & 3.8 & 6 & 6.6 \\
Refusals & 18 & 12.6 & 4 & 7.5 & 5 & 5.5 \\
Nonvalid responses & 17 & 11.9 & 2 & 3.8 & 3 & 3.3 \\
Valid responses & 53 & 37.1 & 31 & 58.5 & 50 & 54.9 \\
\hline Total & 143 & 100 & 53 & 100 & 91 & 100 \\
\hline Attrition & \multicolumn{2}{|c|}{$62.1 \%$} & $36.7 \%$ & \multicolumn{2}{|c|}{$37.5 \%$} \\
\hline
\end{tabular}


The primary cause of attrition was the inability to locate or recontact first-wave participants, especially in the main panel sample (Table 3). Noncontact was the reason for attrition in 36.4 percent of the cases in the second wave of the main panel. This percentage was reduced to 22.6 percent in the third wave of the main panel. The percentage of attrition due to noncontact in the refreshment sample was 24.2 percent. The fact that initial respondents did not know that they were to be contacted again could explain the high percentage of noncontacts.

The composition of dropouts was nonrandom. Females, younger respondents, and those with car availability were less likely to abandon the panel. Panelists having a medium level of education and those who belonged to a nonmotorized household were more likely to abandon the panel (Table 4).

Table 4. Individual Retention Status by Demographics and Socioeconomic Characteristics, Waves 1 and 2

\begin{tabular}{|c|c|c|c|c|c|c|}
\hline & \multicolumn{2}{|c|}{ Stayers } & \multicolumn{2}{|c|}{ Dropouts } & \multicolumn{2}{|c|}{ Total } \\
\hline & $n$ & $\%$ & $n$ & $\%$ & $n$ & $\%$ \\
\hline \multicolumn{7}{|l|}{ Gender } \\
\hline Males & 9 & $17.0 \%$ & 20 & $22.2 \%$ & 29 & $20.3 \%$ \\
\hline Females & 44 & $83.0 \%$ & 70 & $77.8 \%$ & 114 & $79.7 \%$ \\
\hline \multicolumn{7}{|c|}{ Level of education } \\
\hline Low & 18 & $34.9 \%$ & 25 & $27.2 \%$ & 43 & $30.1 \%$ \\
\hline Medium & 17 & $32.6 \%$ & 42 & $46.4 \%$ & 59 & $41.3 \%$ \\
\hline High & 17 & $32.5 \%$ & 24 & $26.4 \%$ & 41 & $28.7 \%$ \\
\hline \multicolumn{7}{|l|}{ Activity } \\
\hline Employed & 32 & $60.5 \%$ & 54 & $59.9 \%$ & 86 & $60.1 \%$ \\
\hline Studying & 17 & $32.6 \%$ & 30 & $33.0 \%$ & 47 & $32.9 \%$ \\
\hline Others & 4 & $6.9 \%$ & 6 & $7.0 \%$ & 10 & $7.0 \%$ \\
\hline \multicolumn{7}{|l|}{ Age } \\
\hline$<26$ & 23 & $43.4 \%$ & 35 & $38.9 \%$ & 58 & $40.6 \%$ \\
\hline $26-35$ & 12 & $22.6 \%$ & 21 & $23.4 \%$ & 33 & $23.1 \%$ \\
\hline $36-45$ & 8 & $15.1 \%$ & 16 & $17.8 \%$ & 24 & $16.8 \%$ \\
\hline$>45$ & 10 & $18.9 \%$ & 18 & $20.0 \%$ & 28 & $19.6 \%$ \\
\hline \multicolumn{7}{|l|}{ Cars } \\
\hline 0 & 7 & $13.2 \%$ & 23 & $25.6 \%$ & 30 & $21.0 \%$ \\
\hline 1 & 29 & $54.7 \%$ & 46 & $51.1 \%$ & 75 & $52.4 \%$ \\
\hline$>1$ & 17 & $32.1 \%$ & 21 & $23.3 \%$ & 38 & $26.6 \%$ \\
\hline \multicolumn{7}{|c|}{ Car availability } \\
\hline yes & 18 & $34.0 \%$ & & & 39 & $27.1 \%$ \\
\hline no & 35 & $66.0 \%$ & & & 104 & $72.9 \%$ \\
\hline
\end{tabular}




\section{Measurement Errors}

Measurement of errors can be divided into two sources (van Wissen and Meurs 1989): within-wave bias (completion diary fatigue effect) and between wave bias (panel effect). The former is due to fatigue effects caused by the four-day trip diary completion. The later is related to reporting errors that occurred over the waves such as panel effects, long-term fatigue effects, etc.

A reduction in daily ST-B use provided by respondents as the trip diary went on was observed (Figure 2). Except for Mondays, the maximum value was always estimated for the first completion day.

\section{Figure 2. Estimation of Daily Average ST-B Use by Completion Day of the Trip Diary and Completion of Initial Day in the First Wave}

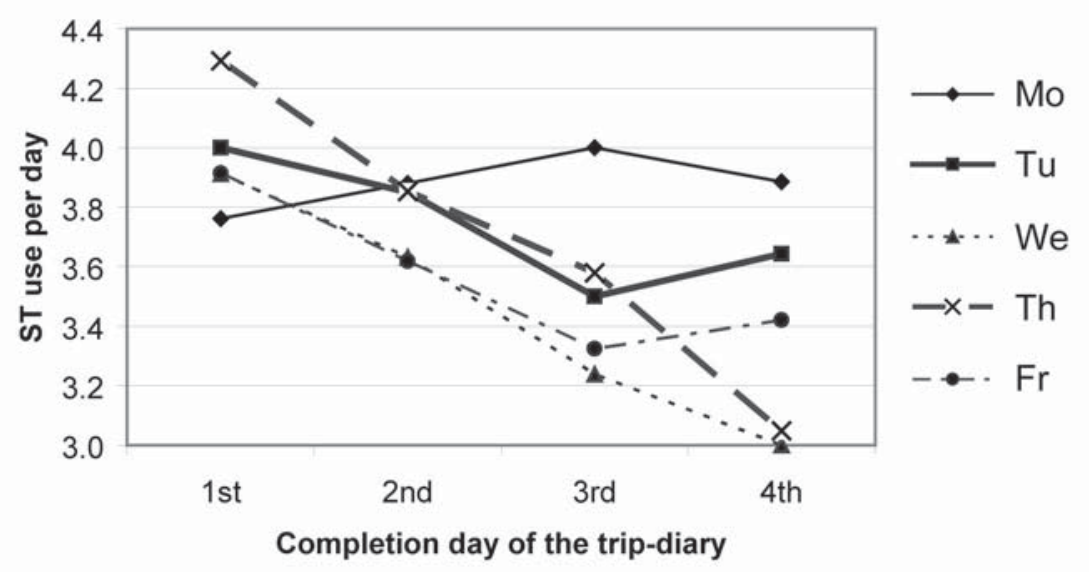

The panel effect in Wave 2 could be evaluated through a comparison between data from panelists who participated in both survey waves and data from the refreshment sample. Before doing this comparison, it was necessary to correct the data from new respondents in Wave 2 for diary fatigue and demographic and socioeconomic differences. 


\section{Correction Procedures}

Correction factors took into account intrawave respondent fatigue effects, changing demographic and socioeconomic distributions of the population and correcting nonrandom attrition.

Firstly, weighting factors were determined for correcting respondent fatigue caused by completion of the four-day trip diary in both the first and second survey waves of panelists and in the refreshment sample data. Different factors were estimated for each completion day, comparing the mobility response for that day in relation to that observed on the first. As a result of this process, an increase of about 10 percent was obtained for the first survey wave (Table 5). It was not possible for the bias in the second survey wave to be analyzed separately for the panel and the refreshment samples ,because of the small size of the former. Thus, common correction factors for both survey waves were evaluated. Because of their application, a 9 percent increase in ST-B use for the second survey wave was obtained in both panelists and refreshment data.

\section{Table 5. Results from Application of Correction Factors in Weekday ST-B Use}

\begin{tabular}{|c|c|c|c|c|c|c|c|}
\hline & & \multicolumn{2}{|c|}{$\begin{array}{c}\text { Average } \\
\text { Observed Values }\end{array}$} & \multicolumn{2}{|c|}{$\begin{array}{c}\text { After Diary Fatigue } \\
\text { Correction }\end{array}$} & \multicolumn{2}{|c|}{$\begin{array}{c}\text { After } \\
\text { Demographic/Attrition } \\
\text { Correction }\end{array}$} \\
\hline & & $S T-B$ Use & $n$ & $S T-B$ Use & $n$ & $S T-B$ Use & $n$ \\
\hline $\begin{array}{l}\overline{\bar{\Xi}} \\
\text { Еू }\end{array}$ & Wave 1 & 3.801 & 250 & 4.193 & 250 & 4.268 & 250 \\
\hline$\frac{5}{\pi}$ & Wave 2 & 3.662 & 141 & 3.988 & 141 & 4.030 & 141 \\
\hline 离 & Wave 2 & 3.760 & 154 & 4.095 & 154 & 4.554 & 154 \\
\hline
\end{tabular}

Secondly, correction factors for adjusting demographic and socioeconomic distributions to match the population were applied to both Wave 1 data from panelists and Wave 2 data from the refreshment sample. An increase of 1.8 percent in ST-B was estimated for the main panelists in Wave 1 and a higher augment of 11.2 percent was estimated for the refreshment respondents in Wave 2. 
Correction for nonrandom attrition in Wave 2 data from the main panelists was carried out assuming that both groups (stayers and dropouts) had similar mobility changes. Following Meurs et. al. (1987), ST-B use in the second wave was estimated for the dropout subsample. The effect of this correction was a slight increase of 1.1 percent in ST-B use.

Finally, the average ST-B use in Wave 2 in the refreshment sample was 13 percent higher than the use from the panelist sample. This difference can be explained by panel effects.

The most important result from the application of the correction procedures is that corrected ST-B use was higher in Wave 2 than in Wave 1, contrary to what was observed for noncorrected values. The aggregate increase in ST-B use was about 6.7 percent from 4.268 ST-B uses in weekdays in Wave 1 to 4.554 ST-B uses in weekdays in Wave 2.

\section{Analysis of Survey Results}

Table 6 presents a summary of ST-B use of the panel over the first and second survey waves. It shows the average ST-B use and the percentage of ST-B use in zone A per weekday per person, by demographic and socioeconomic characteristics for Waves 1 and 2.

Table 6 presents two key points:

1. Average ST-B use per weekdays per person increased from 4.268 to 4.546 ST-B use per weekday (a $6.5 \%$ increase).

2. The A-zone share (ST-B use in the city of Valencia) also increased from 42.9 percent to 46.0 percent.

The panel data analysis reveals that the strongest increase in ST-B use took place among those more than 45 years old, followed by male users, travelers with more than one car at home, and those who claimed to have a car available. In a study of Melbourne (Richardson et al. 1998), it was also found that males tend to use their tickets more, especially older males. In addition, Axhausen et al. (2001) found that public transportation grows jointly with car usage. Travelers with car availability, ST-B users with no car at home, and males also presented high increases of around 15 percent. 
Journal of Public Transportation, Vol. 7, No. 4, 2004

Table 6. Average ST-B Use per Weekday per Person for Waves 1 and 2 by Demographic and Socioeconomic Characteristics

\begin{tabular}{|c|c|c|c|c|c|c|}
\hline & & & & & & \\
\hline & Observations & $S T-B$ Use & $\begin{array}{c}\% \text { in } \\
\text { A-zone }\end{array}$ & $S T-B$ Use & $\begin{array}{c}\% \text { in } \\
\text { A-zone }\end{array}$ & Variation \\
\hline Gender & & & & & & \\
\hline Males & 24 & 3.537 & $28.3 \%$ & 4.271 & $29.2 \%$ & $20.8 \%$ \\
\hline Females & 117 & 4.252 & $45.5 \%$ & 4.612 & $52.2 \%$ & $8.5 \%$ \\
\hline Level of education & & & & & & \\
\hline Low & 49 & 4.281 & $56.3 \%$ & 4.894 & $61.2 \%$ & $14.3 \%$ \\
\hline Medium & 52 & 3.949 & $30.3 \%$ & 3.873 & $38.4 \%$ & $-1.9 \%$ \\
\hline High & 50 & 4.451 & $30.6 \%$ & 4.810 & $35.2 \%$ & $8.1 \%$ \\
\hline Activity & & & & & & \\
\hline Employed & 85 & 4.401 & $30.7 \%$ & 4.448 & $37.8 \%$ & $1.1 \%$ \\
\hline Students & 46 & 4.032 & $33.5 \%$ & 4.448 & $37.8 \%$ & $10.3 \%$ \\
\hline Others & 10 & 3.991 & $88.1 \%$ & 6.151 & $71.1 \%$ & $54.1 \%$ \\
\hline Age & & & & & & \\
\hline$<26$ & 61 & 4.191 & $41.3 \%$ & 4.542 & $47.4 \%$ & $8.4 \%$ \\
\hline $26-35$ & 32 & 4.046 & $22.5 \%$ & 4.157 & $27.3 \%$ & $2.7 \%$ \\
\hline $36-45$ & 21 & 4.476 & $50.2 \%$ & 4.957 & $56.4 \%$ & $10.7 \%$ \\
\hline$>45$ & 27 & 3.902 & $65.2 \%$ & 4.738 & $66.8 \%$ & $21.4 \%$ \\
\hline Cars in household & & & & & & \\
\hline 0 & 19 & 4.533 & $75.0 \%$ & 5.185 & $81.6 \%$ & $14.4 \%$ \\
\hline 1 & 77 & 4.079 & $38.1 \%$ & 4.224 & $45.7 \%$ & $3.6 \%$ \\
\hline$>1$ & 45 & 4.089 & $36.4 \%$ & 4.858 & $38.1 \%$ & $18.8 \%$ \\
\hline Car availability & & & & & & \\
\hline yes & 48 & 4.103 & $36.9 \%$ & 4.808 & $39.2 \%$ & $17.2 \%$ \\
\hline no & 93 & 4.163 & $45.9 \%$ & 4.424 & $53.7 \%$ & $6.3 \%$ \\
\hline Total & 141 & 4.268 & $41.6 \%$ & 4.554 & $48.5 \%$ & $6.7 \%$ \\
\hline
\end{tabular}

The same groups with the highest increases in ST-B use matched those with the lowest changes in zone-use share. Only older travelers used the ST-B mostly in Azone, while the other groups were mostly B-zone users.

On the other hand, ST-B employed users, travelers between 26 and 35 years old, and those with only one car at home presented the lowest increases in ST-B use. In addition, participants with a medium level of education reduced their ST-B use. Middle-aged travelers also presented the lowest usage rates of STs in ticket usage rates in Melbourne (Richardson et al., 1998).

Travelers with a medium level of education, those with no car available, users who had only one car at home, and employed people showed the highest increases in A-zone use of ST-B. The majority of groups with the highest increases in ST-B use in 
A-zone matched with those having the lowest augments in ST-B use. Only travelers who had no car available showed a moderate increase in total ST-B use and a relatively strong change in zone share.

\section{Conclusions and Future Research}

The results of the study demonstrate the feasibility of panel surveys for estimating the evolution of ST use and for identifying behaviors and characteristics of ST users. Transportation policy measures, such as defining new STs or changing current fares for specific users, can be developed more easily using panel data information.

The high attrition rates observed in the panel survey were partially due to the fact that respondents in the first survey wave did not know that they were to be contacted again as members of a panel survey. Additionally, no maintenance technique was used to reduce attrition rates. Therefore, a better recruitment process of panelists and the use of some methods to maintain respondent interest are needed for future panel surveys.

Results of this panel survey indicate that the aggregate increase in ST type-B use was about 6.7 percent from 4.268 uses in weekdays in Wave 1 to 4.554 uses in weekdays in Wave 2. This increase was higher among users more than 45 years old, followed by male users, travelers with more than one car at home, and those who claimed to have car availability. On the other hand, ST-B employed users, travelers between 26 and 35 years old, and those with only one car at home presented the lowest increase in ST-B use. Also, only those with a medium level of education reduced their ST-B use. Car availability and level of education appeared to be statistically significant in a linear regression model. Both of these are positively related to the increase of ST-B use.

Higher initial sample sizes and a greater number of waves are required to achieve more consistent data on the evolution of ST use and to learn more about the individual behavior of ST users. This will enable the study of nonlinear relationships between change of ST use and demographic and socioeconomic explanatory variables. Through the use of dynamic models, it will be possible to simulate their observed behavior and to assess the impact of new fare systems or other transportation supply characteristics in public transport patronage. 
Journal of Public Transportation, Vol. 7, No. 4, 2004

\section{Acknowledgments}

The author would like to thank two anonymous referees for their valuable comments on an early draft of this paper. 
Panel Survey Approach

\section{References}

Axhausen, K. W., A. Simma, and T. Golob, T. 2001. Pre-commitment and usage: Season tickets, cars and travels. European Research in Regional Science 11:101-110.

Blackman, D. 2003. Marketing matters. Surveyor 190 (5728): 16-17.

Duncan, G. J., F. T. Juster, and J. N. Morgan. 1987. The Role of Panel Studies in Research on Economic Behaviour. Transportation Research 21a (4/5), 249263.

Goodwin, P. B. 1997 Have panel surveys told us anything new? In Panels for Transportation Planning, eds T. F. Golob, R. Kytamura and L. Long. Kluwer Norwell, MA: Academic Publishers.

Gründel, T. 2002. The electronic fare as part of the "Intermobil Region Dresden" project: A fare model for electronic fare management within PT. Public Transport International 51 (5): 48-52.

Hensher, D. A. 1986. Longitudinal surveys in transport: An assessment. In New survey methods in transport, eds. E. Ampt, W. Brog, and A. J. Richardson. Utrech: VNU Science Press, 77-98.

Kitamura, R. 1990. Panel analysis in transportation planning: An overview. Transportations Research 24a (6): 401-415.

Knutsson, S. 2003. Valuing rider quality in Swedish special transport services. New findings. Journal of Public Transportation 6 (3): 65-83.

Meurs, H., P. van de Mede, J. Visser, J., and L. van Wissen. (1987). Analyses of Panel Data. Proceedings of the Round Table Conference on the Longitudinal Travel Study. Netherlands Ministry of Transport and Public Works, Project Bureau for Integrated Transport Studies. The Hague.

Miller, S. 2002. A participatory approach to developing new transit services: The city of Phoenix neighborhood circulator planning process. Bus a Paratransit Conference Proceedings. Minneapolis, Minnesota.

Moser, C. A., and G. Kalton. 1979. Survey methods in social investigation, 2nd. ed. Heinemann Educational Books: London.

Polzin, S. E., and M. R. Baltes. 2002. Bus rapid transit: A viable alternative? Journal of Public Transportation 5 (2): 47-69. 
Journal of Public Transportation, Vol. 7, No. 4, 2004

Purvis, C. L., and T. Ruiz. 2003. Standards and practice for multi-day and multiperiod surveys. In Transport Survey Quality and Innovation, eds. P. Stopher and P. Jones, Elsevier, England.

Richardson, A. J., E. Ampt, and A. Meyburg. 1995. Survey methods for Transport Planning, Eucalyptus Press, Melbourne.

Richardson, A. J., P. L. Harbutt, and C. Lester. 1998. Public transport ticket usage surveys-A methodological design. Forum Papers, 22nd Australasian Transport Research Forum, Sydney, Vol. 2, pp. 697-712.

Ruiz, T., M. Fiore, and C. Bayarri. 2003. Survey design for studying the use of the season travel ticket in Valencia, Spain. Transportation Research Board, 82nd Annual Meeting, Compendium of Papers CD-ROM.

Turley, S. K. 1999. A case of response rate success. Journal of the Market Research Society 41 (3): 301-309.

Van Wissen, L. J. G. and H. J. Meurs. 1989. The Dutch mobility panel: Experiences and evaluation. Transportation 16: 99-119.

Watts, B. and A. Ontiveros. 2001. A light rail project that mirrors its community. Rail Transit Conference Proceedings. Boston, Massachusetts.

\section{About the Author}

TomÁs RuIz (truizsa@tra.upv.es) is a lecturer in the transport department at the Technical University of Valencia. Prior to his employment with the university, Dr. Ruiz was a consultant at Estudios, Proyectos y Planificación S.A (EPYPSA).

Dr. Ruiz received a master's degree in civil engineering and a doctorate degree in transportation planning from the Technical University of Valencia. His specific area of doctoral study was personal mobility in urban areas using panel surveys. 